\title{
Imaging of a Giant Frontal Sinus Mucocele with Orbital Displacement: A Case Report
}

\author{
Manvendra Singh ${ }^{1}$, Kamal Kumar Sen ${ }^{2}$, Girendra Shankar ${ }^{1}$, Sudhansu Sekhar Mohanty ${ }^{3}$ \\ ${ }^{1}$ Junior Resident, Department of Radio-diagnosis, Kalinga Institute of Medical Sciences, KIIT University, Bhubaneswar, \\ Odisha, ${ }^{2}$ Professor and Head of the Department of Radio-diagnosis, Kalinga Institute of Medical Sciences, KIIT University, \\ Bhubaneswar, Odisha, ${ }^{3}$ Assistant Professor, Department of Radio-diagnosis, Kalinga Institute of Medical Sciences, KIIT \\ University, Bhubaneswar, Odisha, India.
}

Corresponding author: Sudhansu Sekhar Mohanty, Department of Radio-diagnosis, Kalinga Institute of Medical Sciences, India

DOI: http://dx.doi.org/10.21276/ijcmsr.2019.4.4.28

How to cite this article: Manvendra Singh, Kamal Kumar Sen, Girendra Shankar, Sudhansu Sekhar Mohanty. Imaging of a giant frontal sinus mucocele with orbital displacement: a case report. International Journal of Contemporary Medicine Surgery and Radiology. 2019;4(4):D117-D120.

\section{A B S T R A C T}

A mucocele of a para-nasal sinus is a cyst like expansile and destructive lesion with accumulation of mucoid secretion and desquamated epithelium within. The mass may mimic a primary benign growth in the orbit if the cyst invades the adjacent orbit and continues to expand within the orbital cavity. The frontal sinus is most commonly involved, and since its' floor is shared with the superior orbital wall, there is generally an early displacement of orbit in unmanaged enlarging frontal mucoceles.The diagnosis of a mucocele is based on the history, physical examination, and radiologic findings. An improper management could lead to recurrence of the mass.

Keywords: Frontal Mucocele, Orbital complications, Imaging

\section{INTRODUCTION}

Mucocoeles of the paranasal sinus are regarded as cyst like expansile, destructive lesions with displacement of surrounding structures. The proximity of mucocoeles to the brain is a major concern, if left alone without intervention at the appropriate time. Obstruction of natural ostia which impairs the drainage of Sinus is invariably seen. As a consequence oflong term progressive cystic collection, erosion and invasion into the neighbouring structures viz. nose, orbit, intracranial extension or skin. ${ }^{1,2}$ Multiple aetiopathogenesis have been associated with sinus osteum obstruction caused by the conditions like inflammation, allergy, trauma, anatomic distortions, previous surgery, fibrous dysplasia, osteoma, or ossifying fibroma. ${ }^{2-4}$ Mucoceles of the paranasal sinuses can spread both intra-orbitally and intra-cranially, ${ }^{4,5}$, since the sinuses are in close proximity to the orbit and brain. Of all Paranasal sinuses, frontal sinus and ethmoidal sinus are most often involved (70\% to 90\%). Sphenoid sinus mucoceles are rare. ${ }^{6}$ Mucoceles can form at any age, but the majority are diagnosed in patients beyond fourth decade. Males and females are equally affected. Clinical presentation of the mucoceles varies from asymptomatic to incapacitating headache and visual disturbance. Proptosis (83\%) and diplopia (45\%) are the most common complaints. ${ }^{4}$ We report this case to document the quintessential radiological diagnostic features of giant frontal mucocele causing displacement of the right eyeball.

Various clinical manifestations are seen to be arising due to frontal mucoceles such as proptosis, ptosis, periorbital swelling, restricted ocular movements and inferior displacement of the orbital globe causing diplopia. ${ }^{2}$ The content of the mucocele if infected may cause meningitis or a brain abscess with or without a CSF fistula. The close proximity of paranasal sinus mucoceles to the orbit and skull base predisposes the patient to significant morbidity.

Chronic mucocele shows hyper-intense contents on T1W images with post gadolinium administration enhancement of the wall with intermediate signal contents on T2W images. ${ }^{7,4}$ Differential diagnoses for the soft tissue mass would be dermoid cysts, fungal or tuberculous infection, histiocytosis, fronto-orbital cholesterol granuloma or an uncommon neoplasm..$^{8,9,2,4}$ Computed tomography (CT) and Magnetic Resonance Imaging(MRI) are the modalities of choice for an accurate diagnosis and planning further management. Ultrasonography may be complimentary at times.

\section{CASE REPORT}

A 73-year-old female presented with gradually progressive painless supraorbital, subcutaneous forehead soft mass, gradual diminution of the vision in the right eye and nasal discharge for the past 8 years. The mass showed relatively rapid growth in past 4 months. There was no history of surgery or trauma. The mass was non-tender, inflamed, fluctuant or pulsatile. No focal orbital masses were palpable and cranial nerve examination was normal. Clinical examination of the right eye revealed displacement of orbit and globe temporally and inferiorly with proptosis and mechanical ptosis. Visual 


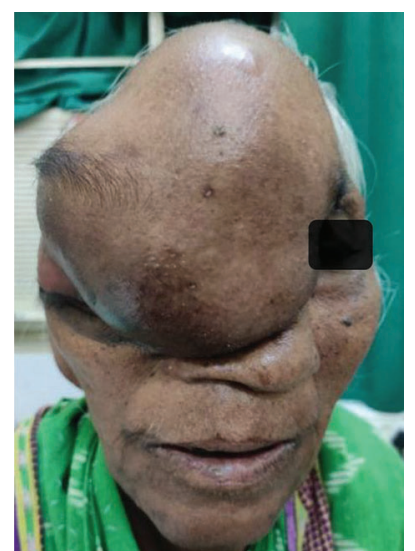

Figure-1: Large soft tissue mass lesion on the forehead extending over the right eye.
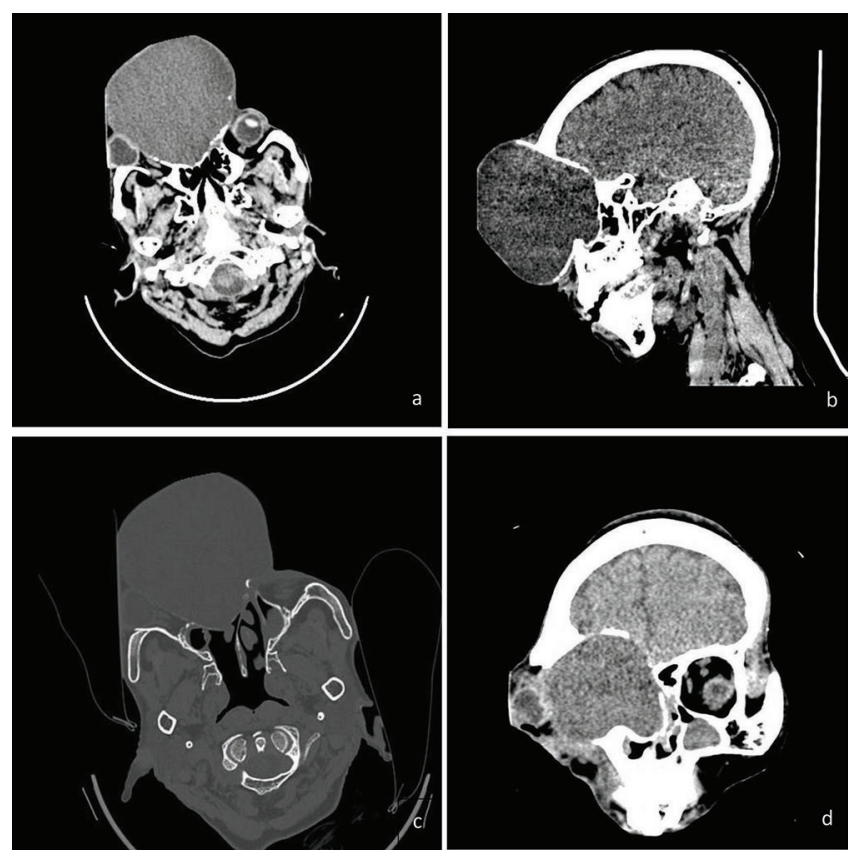

Figure-2: CT Scan, A. Axial, showing well-encapsulated hypodense lesion with peripheral calcified wall, involving right frontal and maxillary sinuses and bilateral ethmoidal sinuses (right>left) pushing the right globe laterally without any intracranial extension. B. Sagittal image showing craniocaudal extension of the lesion and involvement of right frontal and maxillary sinuses. C. Axial- Bone Windowimage showing the thinning and erosion of right maxillary and bilateral ethmoidal (right>left) sinuses. D. Coronal image showing lateral displacement of right eye ball and elevation of floor of anterior cranial fossa.

acuity could not be assessed on the right and was $6 / 24$ on the left.

Computed tomography (CT) scan showed a large (8x $8 \mathrm{x} 7 \mathrm{~cm}$ ) encapsulated extra-axial lesion with CT HU of $[(+10)-(+30)]$, and peripheral calcification along frontal sinus along the superior orbital rim. The lesion was abutting and pushing the right globe laterally maintaining fat plane with it. Superiorly the lesion was indenting and causing resorption and remodelling of frontal bone extending up to the roof. There was no demonstrable evidence of intracranial
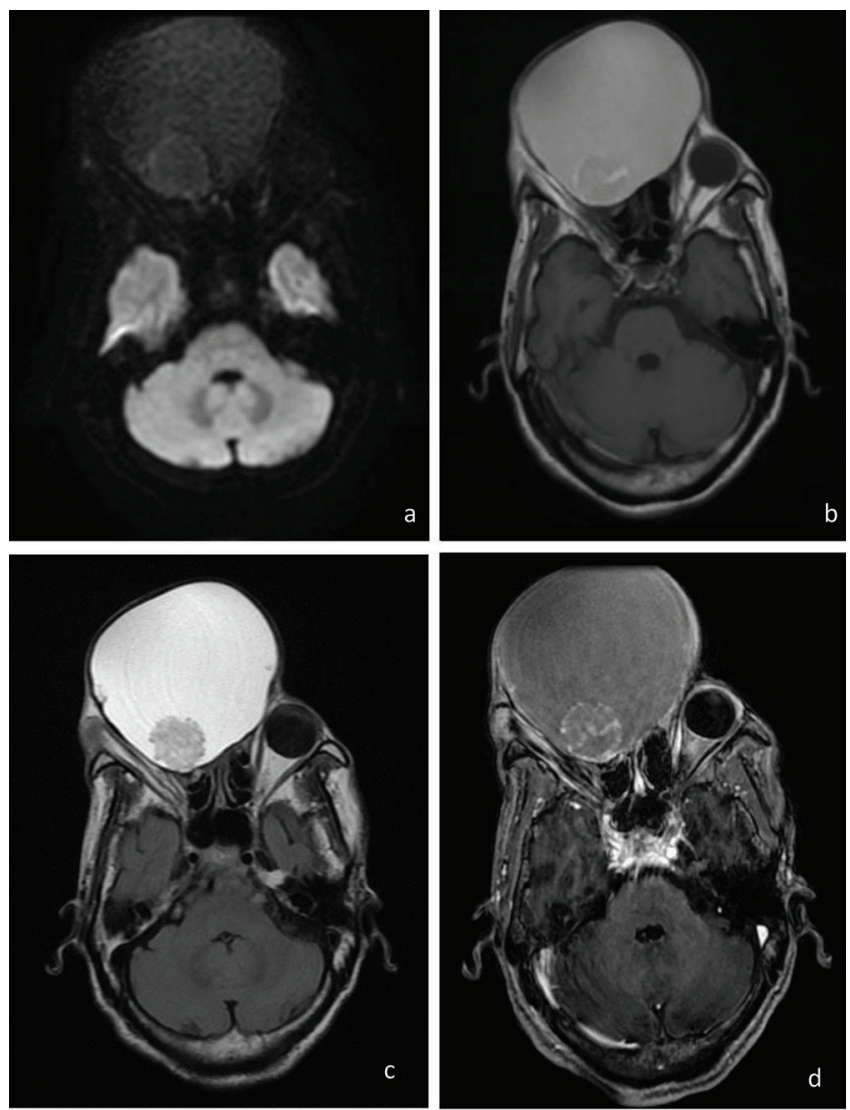

Figure-3: MRI. A. Axial DWI; The lesion showing no diffusion restriction. B. Axial T1; A well-defined large hypointense lesion involving frontal sinus noted on right with a well-defined round hyperintensity within. c. Axial T2 FLAIR; The lesion is hyperintense with a well-defined round hypointensity within likely suggestive of organized hemorrhage. d. Axial post contrast T1; Patchy focal peripheral enhancement of the lesion without any enhancement of the round hyperintense content within.

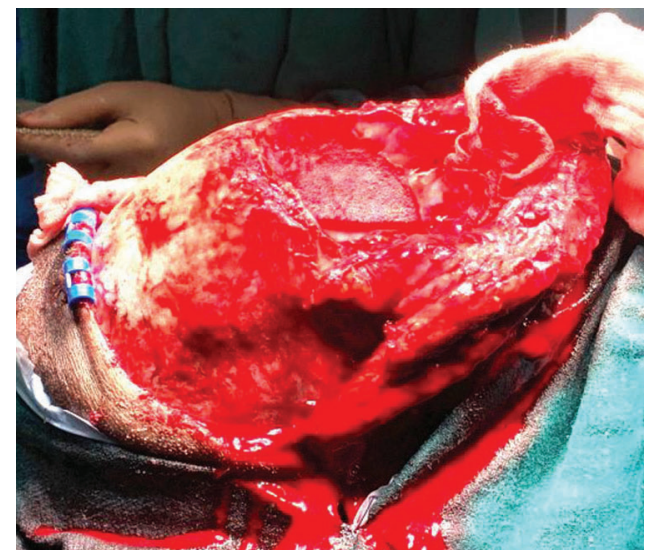

Figure-4: Intra-operative image of everted bony margins and intact floor of mucocele.

extension. Erosion and resorption of the Nasal bone was noted. On MRI, the lesion was hyper intense on T1W/T2W \& FLAIR (Fluid Attenuated inversion recovery) sequences with no diffusion restriction or blooming within as confirmed with susceptibility-weighted (SWAN) imaging. On Fat saturation sequences the lesion washyper intense. There 

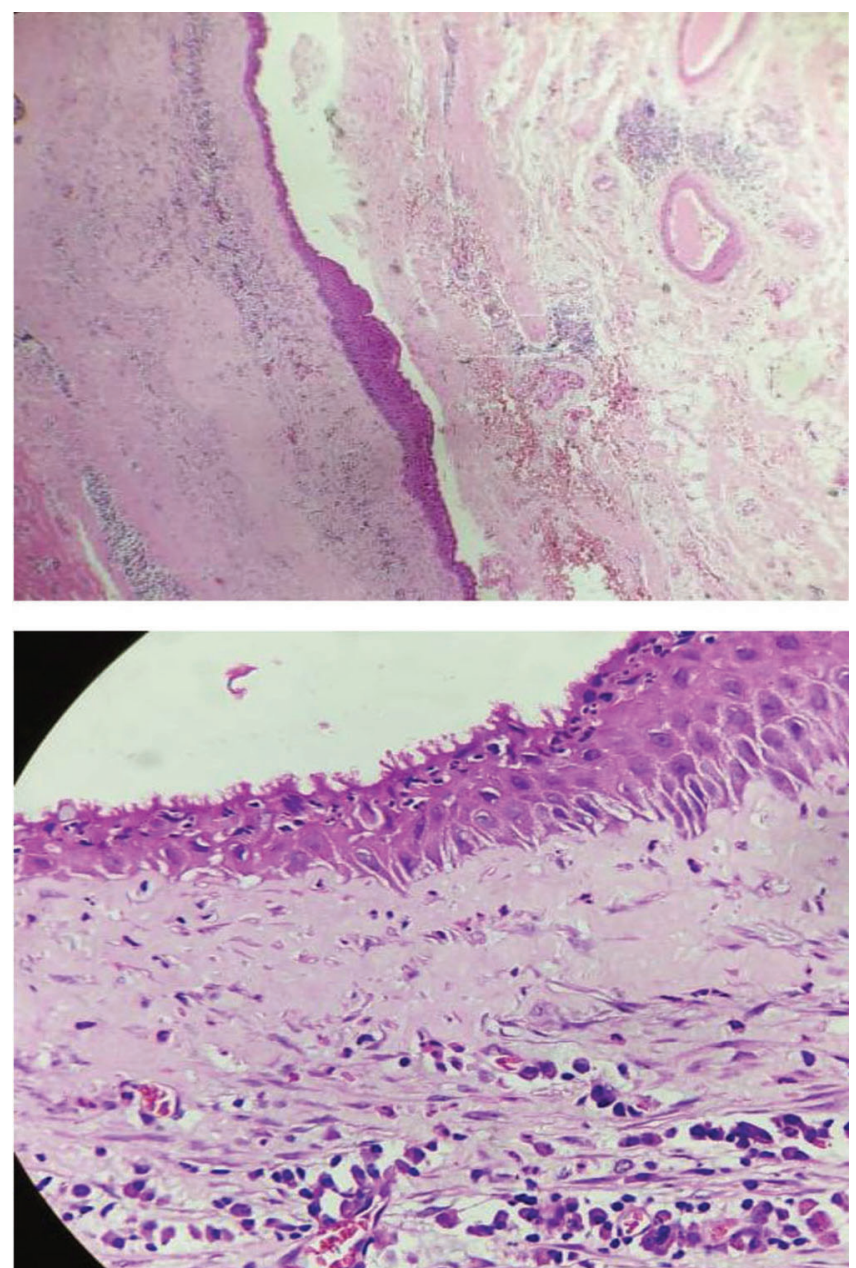

Figure-5: Cyst wall lined by respiratory epithelium with underlying fibrocollagenous tissue and focal metaplasia with diffuse lympho-plasmacytic infiltration are noted on histopathology.

was a T1W heterogeneously hyper intense and T2Wnon enhancing hypointense nodule in the dependent part of the lesionsuggestive of organized haemorrhage /debris. This was found to be mobile on Ultrasound (USG). There was no intracranial extension of the mass. The displaced orbital contents were well delineated.

Surgical removal of the mass with a prosthesis implant was undertaken. The histopathological examination confirmed the radiological diagnosis of mucocele.

\section{DISCUSSION}

Infective, inflammatory, neoplastic or congenital malformations can lead to obstruction of the paranasal sinus ostium and there is gradual accumulation of mucosal secretions ultimately leading to the development of a mucocele. A long standing process leads to distorted perilesional anatomy with soft tissue displacement and bony resorption. ${ }^{7}$

Studies have shown that obstruction and subsequent infection of the frontal sinus leads to localized accumulation of lymphocytes and monocytes which trigger the release of Prostaglandin E2 and collagenase from the surrounding fibroblasts. Elevated levels of Prostaglandin E2 and collagenase have been implicated in the osteolytic process that accompanies the infected mucocele, which translate into the locally aggressive nature of such lesions. ${ }^{8}$

Bony erosions, remodelling, visualisation of anatomical details, the extent of perilesional soft tissue invasion are best studied on $\mathrm{CT}$. ${ }^{9}$ In our case, CT demonstrated expansion and thinning of the bony wall of the frontal sinus with complete erosion of the anterior wall of the Frontal sinus and nasal bone.

Computed tomography (CT) can delineate the expansile homogeneous mass arising from within the sinus with bony remodelling and thinning of the margins with or without marginal sclerosis. However, bone destruction is uncommon. A presence or absence of intracranial extension is also demonstrated. Magnetic resonance imaging (MRI) is superior to CT in the distinction of a mucocele from other soft tissue lesions like neoplasms and recognizing its association with proximate tissues like the brain and orbit. ${ }^{7,9}$ MRI provides a superlative perspective in understanding the nature of such soft tissue cystic lesions and provide differentiation from other soft-tissue neoplasms. In the studied case, the lesion showed T1W/T2W/FLAIR hyperintensity with no diffusion restriction or SWAN blooming with $\mathrm{T} 1$ hyper and $\mathrm{T} 2$ hypointense content due to organised haemorrhage $\&$ debris. Gadolinium enhancement helps characterise the cystic wall anatomy in greater details in chronic stages.

Mucoceles generally tend to be bright on T1W images compared to the brain and iso to hyperintense on T2W images. Neoplastic processes tend to be isointense relative to the brain on both $\mathrm{T} 1$ and $\mathrm{T} 2 \mathrm{~W}$ images. Hyper intensity on T1W images suggests proteinous or hemorrhagic content of a lesion. This may lead to misdiagnosis, hence a dynamic sonographic study is helpful to see the mobility of the content within. One of the other pitfalls of MRI in the diagnosis of mucocoeles is that if it contains inspissated proteinaceous content, it could become almost void of signal on T1W and T2W images, like that of air. MRI alone would be misleading in such cases. On CT, however, the inspissated content would be of high density, making diagnosis straightforward.

The management of Mucoceles is usually surgical, which ranges from functional endoscopic sinus surgery(FESS) to craniotomy, and craniofacial exposure, with or without obliteration of the sinus. ${ }^{6}$ As surgical instrumentation has improved and the pathophysiology is better understood, surgical treatment and endoscopic marsupialisation of mucoceles have evolved into procedures that are less invasive and which emphasize more on surgical drainage over ablation.

\section{CONCLUSION}

The imaging modalities in the diagnosis \& evaluation of Mucocoele invariably would include, CT Scanning and MRI. Ultrasonography may be complimentary. The radiological evaluation provides an insight into the nature and extent of such lesions and help in distinguishing from the differentials. CT and MRI also providesa road map for Surgical, Neuro surgical and maxillofacial repairs for perfect management of such large benign paranasal sinus pathologies. 


\section{REFERENCES}

1. Aggarwal SK, Bhavana K, Keshri A, Kumar R, Srivastava A. Frontal sinus mucocele with orbital complications: Management by varied surgical approaches. Asian Journal of Neurosurgery. 2012;7(3):135.

2. Tan C, Med M, Yong V, Yip L, Med M, Amrith S. An Unusual Presentation of a Giant Frontal Sinus Mucocele Manifesting with a Subcutaneous Forehead Mass. :2.

3. Suri A, Mahapatra AK, Gaikwad S, Sarkar C. Giant mucoceles of the frontal sinus: a series and review. Journal of Clinical Neuroscience. 2004;11(6):214-8.

4. Edelman RR. Clinical Magnetic Resonance Imaging. Saunders Elsevier; 2006. 1275 p.

5. Lund VJ, Milroy CM. Fronto-ethmoidal mucocoeles: a histopathological analysis. J Laryngol Otol. 1991;105(11):921-3.

6. Arrué P, Kany MT, Serrano E, Lacroix F, Percodani J, Yardeni E, et al. Mucoceles of the paranasal sinuses: uncommon location. The Journal of Laryngology \& Otology. 1998;112(9):840-4.

7. Kim Y-S, Kim K, Lee J-G, Yoon J-H, Kim C-H. Paranasal Sinus Mucoceles with Ophthalmologic Manifestations: A 17-year Review of 96 Cases. Am J Rhinol Allergy. 2011;25(4):272-5.

8. Lund VJ, Harvey W, Meghji S, Harris M. Prostaglandin synthesis in the pathogenesis of fronto-ethmoidal mucoceles. Acta Oto-Laryngologica. 1988;106(12):145-51.

9. Tsitouridis I, Michaelides M, Bintoudi A, Kyriakou V. Frontoethmoidal Mucoceles: CT and MRI Evaluation. Neuroradiol J. 2007;20(5):586-96.

Source of Support: Nil; Conflict of Interest: None

Submitted: 21-10-2019; Accepted: 16-11-2019; Published online: 12-12-2019 